\title{
Collection of High Pedigree AM Data for Data Analysis and Correlation
}

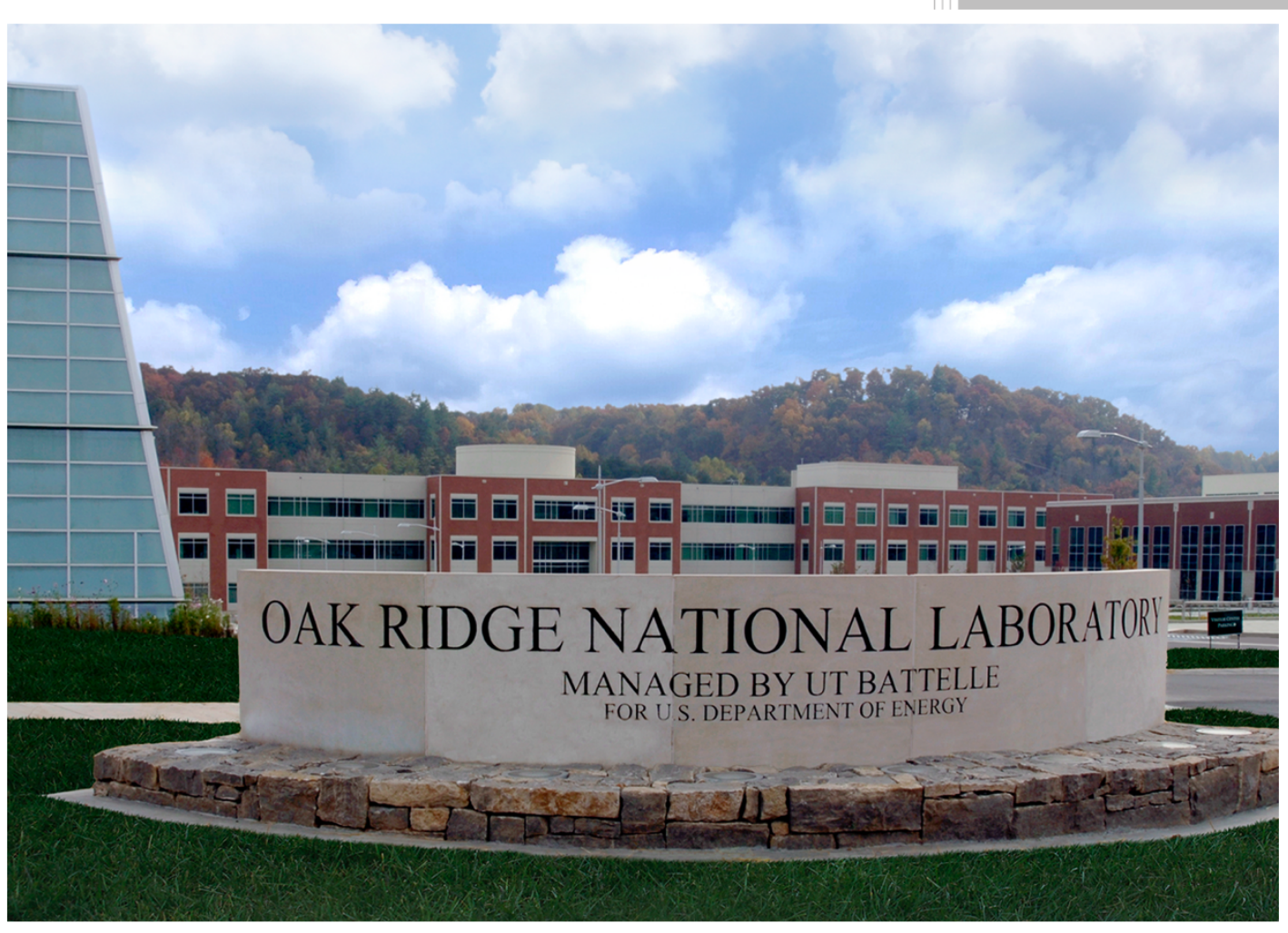

Peeyush Nandwana

October 2019

CRADA FINAL REPORT

NFE-16-06239

Approved for Public Release. Distribution is Unlimited. 


\section{DOCUMENT AVAILABILITY}

Reports produced after January 1, 1996, are generally available free via US Department of Energy (DOE) SciTech Connect.

Website http://www.osti.gov/scitech/

Reports produced before January 1, 1996, may be purchased by members of the public from the following source:

National Technical Information Service

5285 Port Royal Road

Springfield, VA 22161

Telephone 703-605-6000 (1-800-553-6847)

TDD 703-487-4639

Fax 703-605-6900

E-mail info@ntis.gov

Website http://www.ntis.gov/help/ordermethods.aspx

Reports are available to DOE employees, DOE contractors, Energy Technology Data Exchange representatives, and International Nuclear Information System representatives from the following source:

Office of Scientific and Technical Information

PO Box 62

Oak Ridge, TN 37831

Telephone 865-576-8401

Fax 865-576-5728

E-mail reports@osti.gov

Website http://www.osti.gov/contact.html

This report was prepared as an account of work sponsored by an agency of the United States Government. Neither the United States Government nor any agency thereof, nor any of their employees, makes any warranty, express or implied, or assumes any legal liability or responsibility for the accuracy, completeness, or usefulness of any information, apparatus, product, or process disclosed, or represents that its use would not infringe privately owned rights. Reference herein to any specific commercial product, process, or service by trade name, trademark, manufacturer, or otherwise, does not necessarily constitute or imply its endorsement, recommendation, or favoring by the United States Government or any agency thereof. The views and opinions of authors expressed herein do not necessarily state or reflect those of the United States Government or any agency thereof. 
ORNL/TM-2019/1321

CRADA/ NFE-17-06876

Materials Science and Technology Division

Advanced Manufacturing Office

\title{
Collection of Pedigree AM Data for Data Analysis and Correlation
}

\author{
Authors \\ Peeyush Nandwana (ORNL) \\ Ryan Dehoff (ORNL) \\ Zach Simkin (Senvol) \\ Annie Wang (Senvol) \\ Date Published: \\ October 2019 \\ Prepared by \\ OAK RIDGE NATIONAL LABORATORY \\ Oak Ridge, Tennessee 37831-6283 \\ managed by \\ UT-BATTELLE, LLC \\ for the \\ US DEPARTMENT OF ENERGY \\ under contract DE-AC05-00OR22725
}

Approved For Public Release 


\section{CONTENTS}

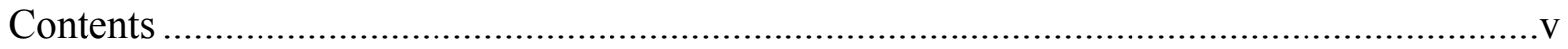

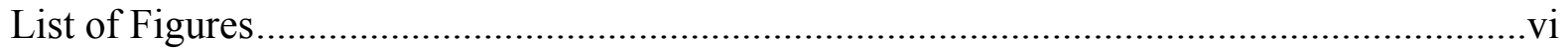

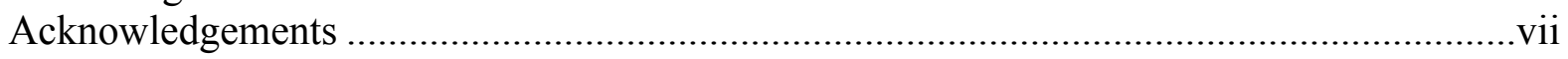

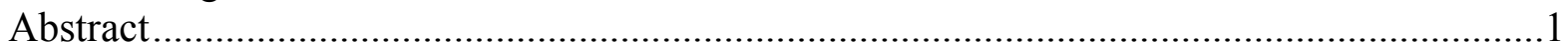

1. Collection of Pedigree AM Data for Analysis and Correlation ........................................1

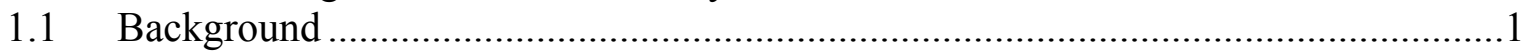

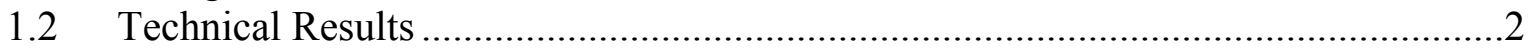

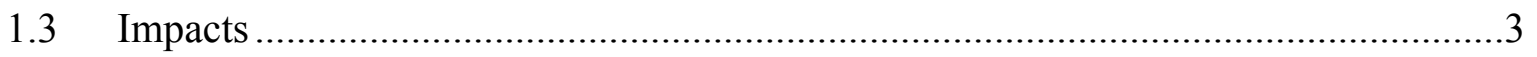

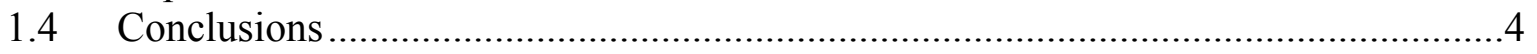

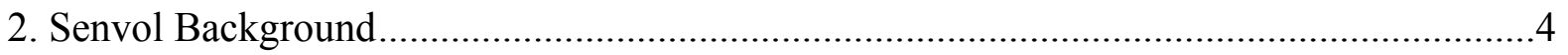




\section{LIST OF FIGURES}

Figure 1. Powder size distribution and the fabricated builds used in the study......................2

Figure 2. Summary of tensile data for the 72 samples tested in the study .............................. 3 


\section{ACKNOWLEDGEMENTS}

This CRADA NFE-17-06876 was conducted as a Technical Collaboration project within the Oak Ridge National Laboratory (ORNL) Manufacturing Demonstration Facility (MDF) sponsored by the US Department of Energy Advanced Manufacturing Office (CPS Agreement Number 24761). Opportunities for MDF technical collaborations are listed in the announcement "Manufacturing Demonstration Facility Technology Collaborations for US Manufacturers in Advanced Manufacturing and Materials

Technologies" posted at http://web.ornl.gov/sci/manufacturing/docs/FBO-ORNL-MDF-2013-2.pdf. The goal of technical collaborations is to engage industry partners to participate in short-term, collaborative projects within the Manufacturing Demonstration Facility (MDF) to assess applicability and of new energy efficient manufacturing technologies. Research sponsored by the U.S. Department of Energy, Office of Energy Efficiency and Renewable Energy, Advanced Manufacturing Office, under contract DEAC05-00OR22725 with UT-Battelle, LLC.

The author PN would like to acknowledge Westmoreland Mechanical Testing and Research, Inc. for their support in the machining and testing of samples. 



\begin{abstract}
ORNL worked with Senvol to develop and evaluate a standard operating procedure for collection of pedigree data for Additive Manufacturing using the Concept Laser XLine 1000r using an Al-Si-Mg alloy. ORNL independently evaluated and provided feedback to Senvol regarding the standard operating procedure document. Those edits were incorporated in the document that was then used to fabricate builds on the Concept Laser XLine 1000r to evaluate the efficacy of the document in collecting pedigreed data. The builds were done with varying build parameters, and the samples were subjected to tensile testing. The tensile data was used as an input for an artificial intelligence-based data analytics framework to determine the correlation between the build parameters and resulting tensile strength.
\end{abstract}

\title{
1. COLLECTION OF PEDIGREE AM DATA FOR ANALYSIS AND CORRELATION
}

This phase 1 technical collaboration project (MDF-TC-2017-129) was started on August 09, 2017 and was completed on August 08, 2019. The collaboration partner Senvol is a small business. In this project, ORNL evaluated a standard operating procedure (SOP) document prepared by Senvol to fabricate aluminum samples via laser powder bed fusion while collecting traceable pedigree data. The builds were fabricated to explore the impact of process parameters on the tensile strength of the resulting material. A correlation analysis was conducted to determine the dependence of tensile strength on these process variables.

\subsection{BACKGROUND}

Senvol is a small business with a focus on being a database and software provider to help companies with additive manufacturing (AM). They have a comprehensive database of various AM machines, processes, and resulting properties. They further provide access to raw pedigreed data to their customers. Additionally, they have developed machine learning software to analyze the vast quantities of data generated by various AM systems across different users. To help companies generate pedigreed data, Senvol has developed a proprietary standard operating procedure (SOP) in an attempt to standardize the operating procedures across various AM systems and users in a way that the data can be imported in their machine learning framework to quickly identify the interdependence of various variables on the resulting microstructures and material properties. This is expected to facilitate rapid development of process parameters for new materials or geometries.

In order to capture information for pedigreed additive manufacturing data (AM), Senvol previously created a proprietary data collection schema that is sent to collaborators when collaborators are building, analyzing, and testing AM builds. These may include things such as the geometric information (build layout, part location in the chamber, spacing between components, etc.), key processing parameters for the AM technology (beam travel speed, beam current, scan pathway, software version, etc.) and any key material testing protocols (sectioning practices, component orientation tracking, mechanical testing configuration, material testing rates, etc.). All of these are critical in terms of understanding the true material response, especially when dealing with multivariate analysis approaches in which several of these variables may be interlinked. The goal of this CRADA was to evaluate Senvol's current best practices document for collecting data to determine if the outlined procedures are adequate for powder bed additive manufacturing technologies.

\subsection{TECHNICAL RESULTS}

In the Phase 1 of this project the machine setup and data collection documentation were reviewed 
and jointly evaluated by ORNL staff and Senvol. The documentation was also compared to current best practices within a research environment (at ORNL MDF). ORNL and Senvol collaborated on determining a test geometry that best captures key features within a build. This geometry was used to evaluate features, such as part location in the build, build orientation, process parameters, etc., that can influence the outcome of build quality. ORNL then oversaw the fabrication of the build and the samples were evaluated based on the specific testing protocols outlined in the documentation.

The data was logged in Senvol's data structure, as detailed in Senvol's current best practices document. Senvol's database was evaluated such that improvements can be made in data collection methods in order to improve the quality of the information extracted from specific builds. This combined Senvol's understanding of industry data collection requirements with the knowledge of a dedicated, experienced user of the technology at ORNL.

ORNL provided critical feedback regarding Senvol's SOP document that was helpful to Senvol for further refining the SOP. As an example, ORNL suggested unifying multiple documents under a single drop down menu and perhaps providing a cloud-based system where pertinent information can be stored under the relevant fields, along with notes from the machine operator, instead of the current format where build logs and operator feedback are saved as separate documents. Another example is that, in the current format, the SOP collects data on powder feedstock as part of the document available to the machine operator, whereas the certificates from the powder supplier are stored in a different location. ORNL suggested that Senvol integrate the two in the form of a software package where the operator can enter the powder conditions and upload the manufacturer's specification documents in the same location. Such feedback is important for Senvol to be able to improve their SOP document and will be critical in its evolution. Owing to the fact that the SOP is a business secret, more details about its nature beyond these examples cannot be publicly provided.

For the current project, Al-10Si-Mg powders were used to make 72 tensile bars that were fabricated by varying laser powder, laser speed, and layer thickness. The samples were then tested for tensile behavior in as-fabricated condition. The powder size distribution and the build layout used for the study is shown in Figure 1.
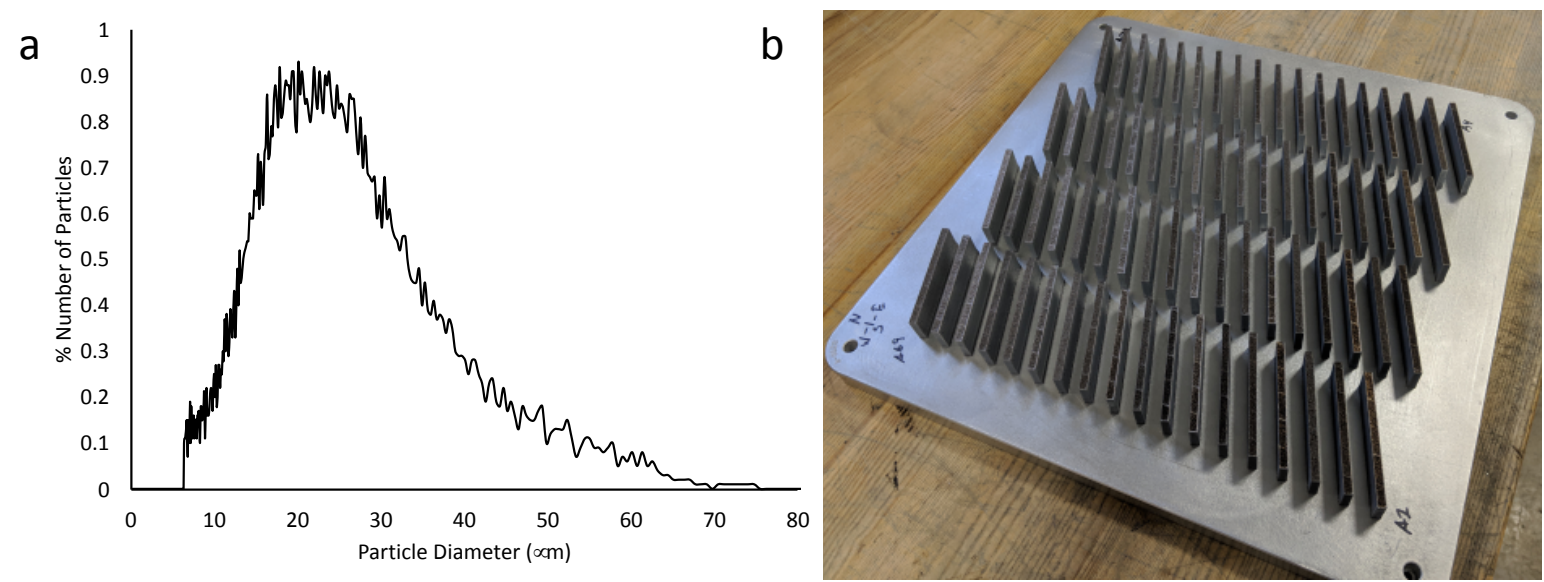

Figure 1. Powder size distribution and the fabricated builds used in the study

The samples were fabricated such that there were two replicates for each condition, i.e. for a given combination of laser power, laser speed, and layer thickness. The detailed process parameters cannot be disclosed owing to Senvol's sensitivity and the competitive advantage that hinges on the data because it directly feeds into their database. The samples were machined into flat dog-bone samples and tested per 
ASTM E-08 standards. Figure 2 shows the plots of yield strength (YS) vs. elongation and ultimate tensile strength (UTS) vs. yield strength (YS).
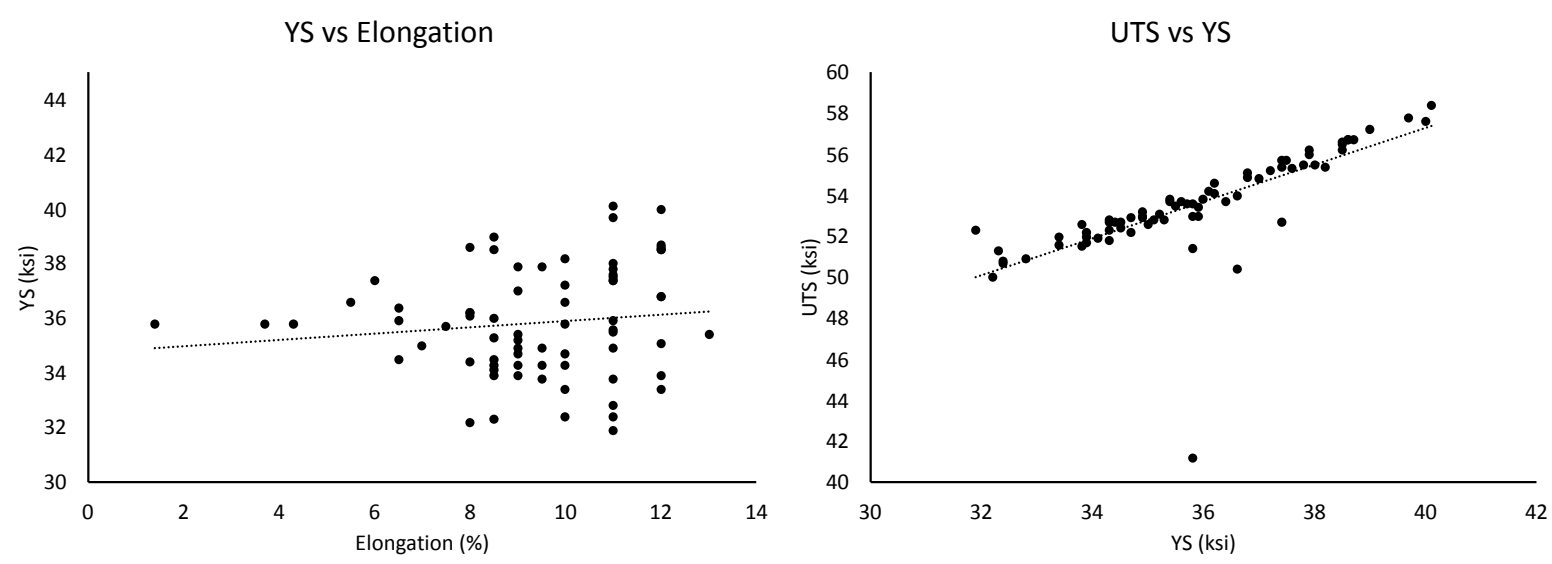

Figure 2. Summary of tensile data for the 72 samples tested in the study

Based on Figure 2, it can be noted that there is a significant scatter in elongation of the samples, whereas the YS correlates linearly with the UTS. Theoretically, in a defect-free material with random texture, YS and elongation are inversely related, i.e. elongation decreases with increasing strength when microstructure is the only dominant variable, and YS and UTS typically increase linearly with refining microstructure. However, in AM, especially in the current case where multiple process parameters are varied, porosity can influence the YS, UTS and elongation in tandem with microstructural features. Usually, porosity is $<1.5 \%$ in most fusion-based processes and may not have a direct impact on the YS and UTS, but can cause noticeable scatter in elongation by being the preferred sites for initiation of failure.

The linear nature of YS vs. UTS plot in Figure 2 is probably indicative of minimal changes in the microstructure resulting from changes in process parameters, whereas the scatter in the YS vs elongation plot is indicative of the dominant role of porosity in governing elongation to failure. Various literature sources in AM also suggest that porosity is sensitive to laser power, speed and layer thickness.

In order to better understand the relationships between the process variables and the resulting tensile behavior, a Spearman rank correlation function was deployed. It was found that the elongation has a weak inverse correlation with layer thickness and a weak direct correlation with the energy density; i.e., elongation reduces with increasing layer thickness and decreasing energy density. This correlation further affirms the hypothesis that the scatter in elongation is due to the presence of porosity because layer thickness affects the energy density and porosity in powder bed fusion processes. The lack of any correlation of YS or UTS with either layer thickness or energy density also indicates that these two properties (YS and UTS) are more dependent on microstructure than on the defect structure. To delineate the effect of porosity and microstructure on YS and UTS, more in-depth microstructural analysis needs to be undertaken, which is beyond the scope of this study.

\subsection{IMPACTS}

The impact of this project will be significant in helping the AM industry understand the necessity of producing pedigree data. Additionally, this project will help uncover the effect of tractability of data, fabrication procedures and testing procedures on the quality of data as it relates to additive manufacturing. In particular, the work from this CRADA allowed Senvol to improve quality of the data being produced from and input into Senvol's products, which have been adopted by industry to evaluate particular 
technologies and materials for AM. With having all of the data put into an appropriate framework, the data can easily be evaluated, manipulated, and utilized by the different industrial sectors utilizing Senvol's products. Evaluation work from this CRADA has allowed Senvol and ORNL to optimize the data architecture such that the quality of knowledge that can be extracted from the datasets is improved. Generating the data at the scale in this work and leveraging the use of correlation functions can provide

the basis for isolating the impacts of different variables on resulting tensile properties. This can be helpful in developing process parameters for new materials, especially as the size of the database increases.

\subsection{CONCLUSIONS}

In conclusion, ORNL and Senvol have demonstrated that pedigree data collection is critical to understanding the quality of AM materials, and ensured that Senvol is capturing all of the nuanced data required to accurately extract information. This will strengthen Senvol's business by ensuring proper data collection methods and improve the quality of Senvol's products: the Senvol Database, Senvol Indexes, and Senvol ML, which is currently under development. Furthermore, this is a single comprehensive study that has explored the impacts of different process variables on the tensile behavior of Al-10Si-Mg alloy fabricated via laser powder bed fusion in depth.

\section{SENVOL BACKGROUND}

Senvol's various products and services allow companies to access, generate, and analyze additive manufacturing data. Senvol's products and services have been used in a variety of Fortune 500 companies and government agencies in industries such as aerospace, oil \& gas, consumer products, and automotive. Senvol's executives have been published in various additive manufacturing industry journals and have been featured speakers at numerous trade shows and conferences. Senvol is a Gold Member of America Makes, a public-private partnership that is comprised of leading organizations in the additive manufacturing industry. Senvol's executives have been appointed to leadership positions through the industry as members of SME's Additive Manufacturing Advisory Board, the ASTM International F42 Committee on Additive Manufacturing Technologies, ASME's Technical Advisory Panel (TAP) for additive manufacturing, and also as Vice Chair of the Data Management Committee for SAE. 